\title{
Meta-analytic review on the impact of temperature and water activity in deoxynivalenol synthesis by Fusarium graminearum
}

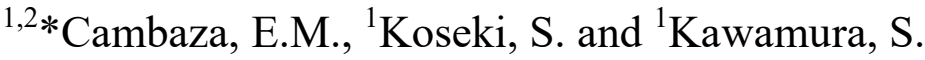 \\ ${ }^{1}$ Laboratory of Agricultural and Food Process Engineering, Graduate School of Agriculture, Hokkaido \\ University, Kita 9, Nishi 9, Kita-ku, Sapporo, Hokkaido 060-8589, Japan \\ ${ }^{2}$ Department of Biological Sciences, Faculty of Sciences, Eduardo Mondlane University, Av. Julius Nyerere, \\ nr. 3453 Maputo, Mozambique
}

\author{
Article history: \\ Received: 28 May 2018 \\ Received in revised form: 27 \\ June 2018 \\ Accepted: 28 June 2018 \\ Available Online: 3 July 2018 \\ Keywords: \\ Water activity, \\ Temperature, \\ Deoxynivalenol, \\ Fusarium graminearum
}

DOI:

https://doi.org/10.26656/fr.2017.2(5).200

\begin{abstract}
Deoxynivalenol (DON) is a mycotoxin produced by Fusarium species, causing involuntary regurgitation in animals including humans. Some studies showed that its synthesis is influenced by environmental factors such as temperature and water activity $\left(\mathrm{a}_{\mathrm{w}}\right)$. This article presents a meta-analysis of two major studies on the phenomenon, discussing on what should be expected in future research in this direction. The specimens were cultured in maize and wheat. In all experiments, Fusarium graminearum was incubated at $15^{\circ} \mathrm{C}, 22^{\circ} \mathrm{C}, 25^{\circ} \mathrm{C}, 28^{\circ} \mathrm{C}$ and $30^{\circ} \mathrm{C}$. At room temperature, the water activity was set to $0.95,0.97,0.99$, and 0.995 . Then, levels of DON were quantified weekly since the 14th day until the 49th day, using HPLC with UV detector at $220 \mathrm{~nm}$. Temperature and water activity seem to have an impact on DON synthesis, though the true correlations still require further analysis.
\end{abstract}

\section{Introduction}

Deoxynivalenol (DON or vomitoxin) is a mycotoxin belonging to the group of sesquiterpenoid epoxytrichothecenes, capable of inhibiting protein synthesis and causing gastrointestinal disorder such as vomit and probably growth impairment in animals and humans (Martins and Martins, 2002; Pestka and Smolinski, 2005). It has been associated to Fusarium head blight (FHB), a common wheat, and barley and maize disease caused by $F$. graminearum, $F$. moniliforme and less frequently by $F$. culmorum, $F$. proliferatum, and $F$. equiseti.

Yoshizawa (2013) published a review of over three decades of research on DON and nivalenol (NIV). He mentioned at least eight FHB outbreaks in Japan between 1890 and 1970, resulting in considerable yield losses of wheat and barley, and several cases of human and animal intoxication. He also mentioned two human intoxications in Hokkaido, in 1956. Weidenbörner (2001) described a simultaneous intoxication with NIV in Japan, 1972. Kubo et al. (2013) described more recent cases (2002 to 2006) where DON was found in more than half of 7746 wheat samples. He said 3.3\% were above the provisional limit regulated for Japan $(1.1 \mathrm{mg} / \mathrm{kg})$.

The most commonly reported detection methods are mass spectrometry, radioimmunoassay (RIA), enzymelinked immunosorbent essay (ELISA), and several forms of chromatography including thin-layer chromatography (TLC), gas chromatography (GC), and high-performance liquid chromatography (HPLC) (Cahill et al., 1999; Pascale and Visconti, 2008). They have been improving overtime and they all have advantages and disadvantages (Pascale and Visconti, 2008). Thus, the best choice might depend on the circumstances and the aim of the entities using them. HPLC is perhaps the most commonly reported in Fusarium toxin studies (Astoreca, 2017).

This study aimed to meta-analyze two key studies on the impact of temperature and water activity on DON synthesis by $F$. graminearum.

\section{Methodology}

The studies analyzed were performed by Martins and Martins (2002), in Portugal, and Marin et al. (1995), in Argentina. The following variables were collected from all authors: growing medium (substrate), $F$. graminearum strain, incubation time, temperature, water activity and DON levels. The data were then submitted to statistical analyses, consisting on tests of hypotheses $(p=0.05)$ and descriptive parameters. 


\section{Results and discussion}

\subsection{DON overall levels}

These studies used natural sources of the nutrient, different from the yeast extract agar. One shall consider the latter as designed to specifically feed the fungi in a balanced fashion. Thus, such appropriated nourishment will reduce the toxin concentration yet increasing the range of incubation time, temperature and $a_{w}$ at which the fungus will produce DON. It might reduce DON content since stress promotes the production of secondary metabolites. The real range of the variables is probably higher because a more nutritious medium also favors the fungus' resistance to harsh environments. Moreover, one shall be aware that different media might make hard the sample to purify for HPLC when compared to others. Figure 1 supports the fact that differences in substrate might affect the mycotoxin synthesis $(p=0.003)$, though there are still other factors to consider such as strain.

The genetic differences might lead a fungus to produce more or less of any secondary metabolite, including mycotoxins. The wild Portuguese strain used by Martins and Martins (2002) and the RC17-2 and RC 22-2 used by the Argentineans authors certainly differ from the Giberella zeae (Schwabe) Petch of the JCM database. Indeed, the meta-comparison of median DON levels shows significant differences between the strains (Figure 2). The wild strain showed the highest value.

Yet, disregarding the differences of media and strains, one shall expect the incubation time, temperature

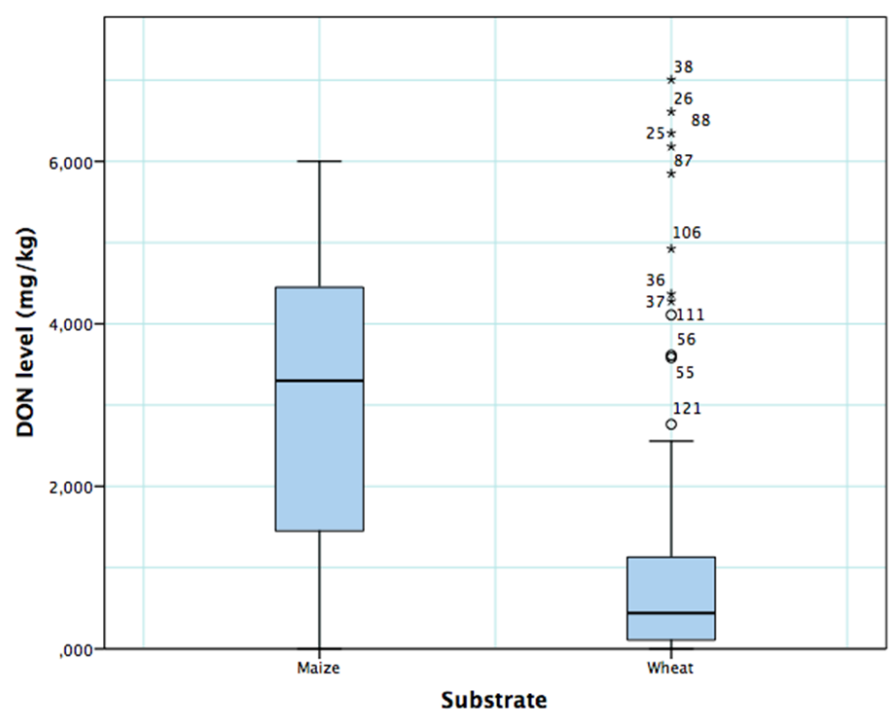

Figure 1. Differences in DON level between $F$. graminearum grown in maize and wheat. The commas (,) separate the decimals. It is represented as commas because the analytical tool is setup in Portuguese system. The data above the right box are values out of the confidence interval. The numbers represent the samples according to the order they were arranged in the spreadsheet used to make this chart. and $\mathrm{a}_{\mathrm{w}}$ to have the same sort of impact over the DON production, either it is subtle or not. An overview of the levels in these studies shows the average to be $1.05 \mathrm{mg} /$ $\mathrm{kg}$, inside a confidence interval (95\%) of $0.7-1.3 \mathrm{mg} / \mathrm{kg}$. A Kolmogorov-Smirnov test revealed a significant difference among the DON levels $(p<0.001)$. Hence, the overall variation resulted in distinct profiles.

As Figure 3 below shows, the incubation time has also shown some association with DON synthesis $(\mathrm{p}=$ $0.001)$. This observation is intuitive since the fungus germinates and accumulates the toxin in the medium, especially when it reaches the "stationary" phase and starts the senescence. However, regression analyses do not show considerable with any polynomial function below the $6^{\text {th }}$ degree, nor with any major algebraic nonparametric function as $\mathrm{R}^{2}$ was consistently lower than 0.5 . Thus, the best fit its behavior is still to be found. Similar regressions were also performed for temperature and $\mathrm{a}_{\mathrm{w}}$, showing the same sort of result $\left(\mathrm{R}^{2}<0.5\right)$.

If one assumes DON synthesis as a function of growth itself, at most directly proportional, one can expect the logistic regression as an applicable fit for both phenomena when in a batch system. However, one has to consider the toxin accumulation as influencing the measurement, probably resulting in accrual values. Anyway, it might not be heavily influencing the system as there was actually a decrease starting from the $35^{\text {th }}$ day of the experiments. One factor affecting the measurement could have been the fact that these authors always took the colonies' oldest parts, usually the ones with the highest toxin concentrations.

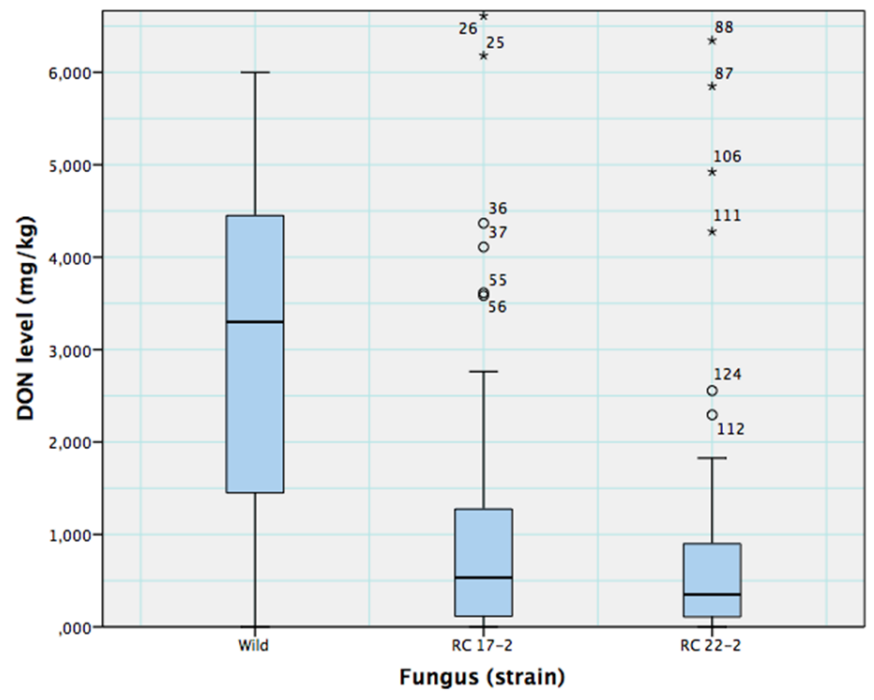

Figure 2. Median values of DON levels between the different $F$. graminearum strains $(\mathrm{p}=0.008)$. The commas (,) separate the decimals. It is represented as commas because the analytical tool is setup in Portuguese system. The data above the boxes are values out of the confidence interval. The numbers represent the samples according to the order they were arranged in the spreadsheet used to make this chart. 


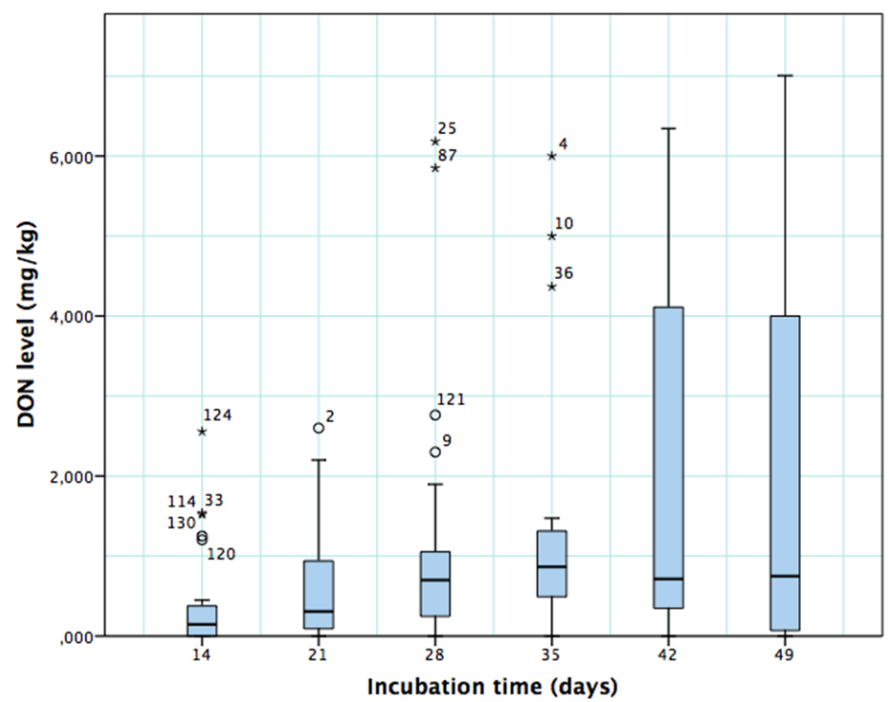

Figure 3. DON level in relation with incubation time. The commas (,) separate the decimals. It is represented as commas because the analytical tool is setup in Portuguese system. The data above the boxes are values out of the confidence interval. The numbers represent the samples according to the order they were arranged in the spreadsheet used to make this chart.

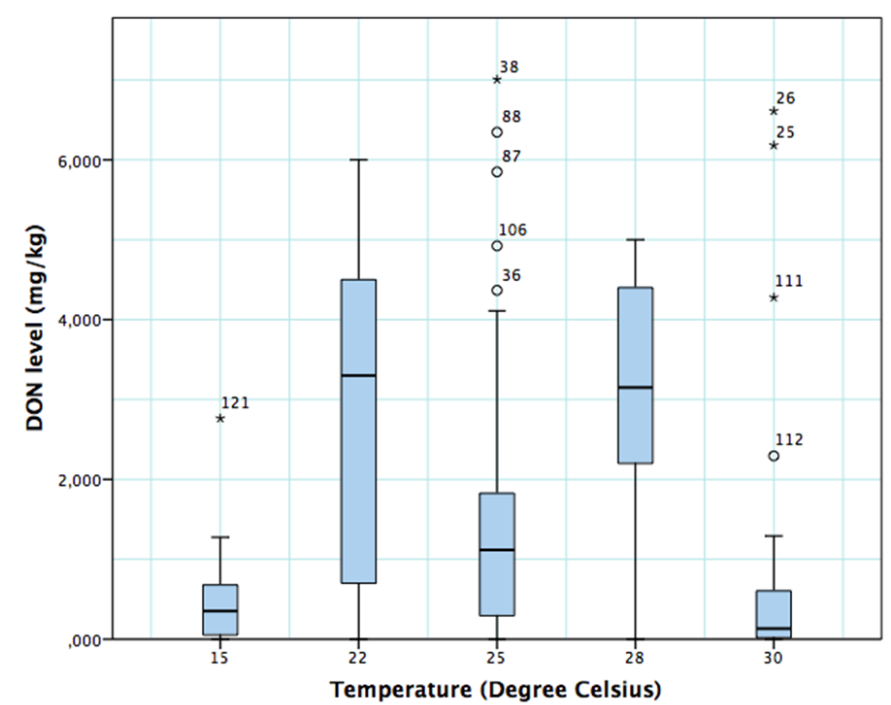

Figure 4. The relationship between temperature and DON level. The commas (,) separate the decimals. It is represented as commas because the analytical tool is setup in Portuguese system. The data above the boxes are values out of the confidence interval. The numbers represent the samples according to the order they were arranged in the spreadsheet used to make this chart.

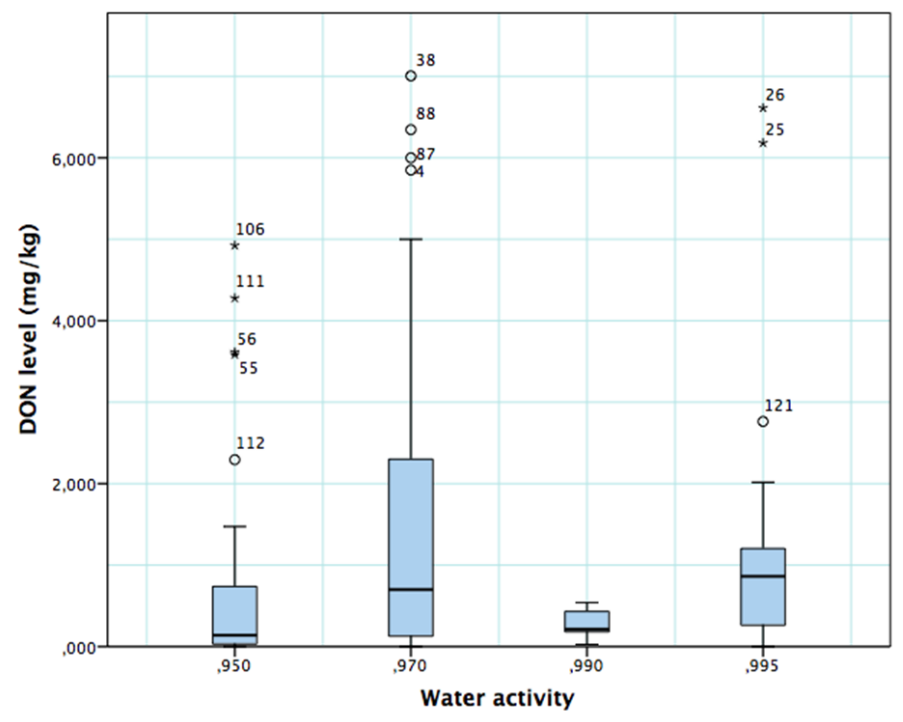

Figure 5. The relationship between water activity and DON level. The commas (,) separate the decimals. It is represented as commas because the analytical tool is setup in Portuguese system. The data above the boxes are values out of the confidence interval. The numbers represent the samples according to the order they were arranged in the spreadsheet used to make this chart.

\subsection{DON and temperature}

Regarding the temperature (Figure 4), a KruskalWallis test suggests it influenced the toxin production ( $\mathrm{p}$ $=0.000)$. Yet, the relationship seems to be complex as the room temperature $\left(25^{\circ} \mathrm{C}\right)$ has shown a probably unexpected low rate if compared to the immediately close values. There are various possible causes for it. It might be related to the discrepancies between the strains. For example, one can notice a wider dispersion as the temperature comes closer to $25^{\circ} \mathrm{C}$, probably the optimal for fungal growth. This dispersion possibly reflects the differences between the way each strain adapts to the medium and produces the toxin. Another possible cause of contamination.
Natural enemies can compete with the fungus and affect its growth, and it would be reasonable that the optimal temperature for fungal growth is as optimal for some of these microorganisms. However, they can hardly compete with $F$. graminearum at less favorable environments. The current experiment will help in understanding better what might have happened.

\subsection{DON and water activity}

Finally, they also analyzed water activity ( $\mathrm{p}=$ 0.026). The highest DON levels were at $a_{w} 0.97$ and 0.995 (Figure 5). If one looks at the boxplot below, easily notice that $\mathrm{a}_{\mathrm{w}}$ of 0.99 has little representativeness within the sample (6 samples of 132) and even shorter 
confidence interval. Thus, the mycotoxin content might well be increasing with $\mathrm{a}_{\mathrm{w}}$. Marin, Sanchis, and Magan (1995) identified 0.98-0.944 as the best range for growth and 0.92 as a harsh environment for it using $F$. moliniforme and $F$. proliferatum. If growth has direct proportionality with mycotoxin production, their result is fairly consistent with these observations. Yet, one cannot ignore the fact that Marin et al. (1995) used different species, though from the same genera.

\section{Conclusion}

The meta-analysis provided us with some ideas of the results one shall expect from the current experiment but one has to be aware of the technical differences.

The temperature and water activity affect the DON level. Extreme temperatures, so as an optimal growth temperature, seem associated to low toxin synthesis, certainly for different reasons: the extreme by not allowing the Fusarium to mature enough to produce the toxin and the optimal by not stimulating the secondary metabolism.

The water activity is probably directly proportional to DON synthesis but it seems at certain extent obscured by other factors such as nutritional and genetic.

Future researchers are recommended to start by using a minimal medium such as yeast extract agar (YEA) because it can prevent any influence of the host organism. Furthermore, these studies should have few standard strains so that they could be properly compared.

\section{Acknowledgments}

Japanese Ministry of Education, Culture, Sports, Science, and Technology (MEXT) for the financial support.

\section{References}

Astoreca, A., Ortega, L., Fígoli, C., Cardós, M., Cavaglieri, L., Bosch, A. and Alconada, T. (2017). Analytical techniques for deoxynivalenol detection and quantification in wheat destined for the manufacture of commercial products. World Mycotoxin Journal, 10(2), 111-120. https:// doi.org/10.3920/WMJ2016.2121

Cahill, L.M., Kruger, S.C., McAlice, B.T., Ramsey, C.S., Prioli, R. and Kohn, B. (1999). Quantification of deoxynivalenol in wheat using an immunoaffinity column and liquid chromatography. Journal of Chromatography A, 859(1), 23-28. https:// doi.org/10.1016/S0021-9673(99)00846-8

Kubo, K., Kawada, N. and Fujita, M. (2013). Evaluation of Fusarium Head Blight Resistance in Wheat and the Development of a New Variety by Integrating Type I and II Resistance. Jarq-Japan Agricultural Research Quarterly, 47(1), 9-19. https:// doi.org/10.6090/jarq.47.9

Marin, S., Sanchis, V. and Magan, N. (1995). Water activity, temperature, and $\mathrm{pH}$ effects on growth of Fusarium moniliforme and Fusarium proliferatum isolates from maize. Canadian Journal of Microbiology, 41(12), 1063-1070. https:// doi.org/10.1139/m95-149

Martins, M.L. and Martins, H.M. (2002). Influence of water activity, temperature and incubation time on the simultaneous production of deoxynivalenol and zearalenone in corn (Zea mays) by Fusarium graminearum. Food Chemistry, 79(3), 315-318. https://doi.org/10.1016/S0308-8146(02)00147-4

Pascale, M. and Visconti, A. (2008). Overview of detection methods for mycotoxins. In Leslie, J.F., Bandyopadhyay, R. and Visconti, A. (Eds.) Mycotoxins: Detection Methods, Management, Public Health and Agricultural Trade, p. 171-183. United Kingdom: CAB International. https:// doi.org/10.1079/9781845930820.0171

Pestka, J.J. and Smolinski, A.T. (2005). Deoxynivalenol: toxicology and potential effects on humans. Journal of Toxicology and Environmental Health, Part B, 8(1), 39-69. https:// doi.org/10.1080/10937400590889458

Weidenbörner, M. (2001). Encyclopedia of Food Mycotoxins. US: Springer-Verlag Berlin Heidelberg. https://doi.org/10.1007/978-3-662-04464-3

Yoshizawa, T. (2013). Thirty-five Years of Research on Deoxynivalenol, a Trichothecene Mycotoxin: with Special Reference to Its Discovery and Cooccurrence with Nivalenol in Japan. Food Safety, 1 (1), 2013002-2013002. https://doi.org/10.14252/ foodsafetyfscj.2013002 